\title{
Guest Editorial: Nanophotonics in Europe
}

\author{
David L. Andrews ${ }^{a}$ and Tom G. Mackay \\ ${ }^{a}$ School of Chemical Sciences, University of East Anglia, Norwich NR4 7TJ, U.K. \\ david.andrews@physics.org \\ bchool of Mathematics, University of Edinburgh, Edinburgh EH9 3JZ, U.K. \\ T.Mackay@ed.ac.uk
}

While nanophotonics is a truly international discipline, its antecedents are firmly rooted in European science and technology. Famously, for example, metallic nanoparticles have been shown to be responsible for the deep red hue of some ancient Roman colored glass vases [1]. Building upon a rich heritage, Europe has more recently played host to many ground-breaking developments in nanophotonics, with wide areas of application. The continent continues to thrive as a major contributor to nanophotonics research, as is highlighted by the papers presented in the following Special Section on Nanophotonics in Europe. It is a collection that offers a representative view of current research in a broad variety of areas of nanophotonics. Both experimental and theoretical works are described, in reviews and original research articles. The papers selected for the Special Section originate either directly from Europe, or as presentations at SPIE Europe's major Photonics Europe conference held in Strasbourg in April 2008. Perhaps it is apt that three of the papers originate from Scotland, the country of birth of James Clerk Maxwell [2] - arguably a founding grandfather of today's nanophotonics.

The Special Section begins with a review, by Dienerowitz, Mazilu and Dholakia, of optical manipulation in the context of nanoparticles [3]. While optical trapping is now wellestablished for micron-sized particles, the trapping and manipulation of nanoparticles represents an exciting new development. In their review the authors discuss both theoretical and experimental aspects, pertaining to dielectric, metallic and molecular nanoparticles. A widespread problem in nanoplasmonics is absorption loss, which causes damping of surface plasmons. As described by Noginov [4] in the second paper, such losses can be compensated by optical gain in a dye-doped dielectric material adjacent to the metal surface. Experimental results are presented which demonstrate this effect in the case of propagating of surface plasmon polaritons, and experimental evidence for the stimulated emission of surface plasmon polaritons at optical frequencies is also presented. A comprehensive review of solid immersion lens microscopy applications in nanophotonics is delivered in the third paper, by Serrels et al. [5]. These applications include semiconductor integrated-circuit inspection and spectroscopy, optical data storage and photolithography. A theoretical analysis of the solid immersion lens is provided, followed by a discussion of the advantages offered by these lenses in the interrogation of photonic and electronic nanostructures. In the fourth paper of the Special Section, Gaburro [6] describes how two complementary principles, namely the Doppler shift and time refraction, may be exploited via time-dependent dielectric boundaries, to achieve shifts in the carrier frequency of an optical pulse without inducing changes in the pulse shape or coherence. These techniques may be applied to generate event horizons, analogous to those arising in gravitational theory. Finite-difference time-domain results are presented, along with some preliminary experimental results. The work typifies the curiosities that are emerging in this field, where the behavior of light is very remote from everyday experience. The fifth paper addresses an already established experimental method - and a key topic in nanophotonics, the controlled delivery of electromagnetic energy at nanometer scales. Here, Vogel and Gramotnev [7] consider the focusing of plasmons using tapered metal rods, motivated by applications in near-field microscopy and spectroscopy, for example. Optimal 
design parameters for the metal rods are determined by a combination of analytical and numerical methods. In the sixth paper, Vincenti et al. [8] propose a novel molecular hydrogen sensor, based on a sub-wavelength slit cut into a thick palladium substrate. The sensor relies upon on enhanced harmonic generation processes, arising from a combination of cavity effects and surface waves inside the slit. Using numerical techniques, the authors demonstrate that nonlinear effects and enhanced transmission are both acutely sensitive to minute quantities of hydrogen absorbed by the palladium substrate. Finally, in the last paper of this Special Section, Mackay [9] describes some analytical extensions to homogenization methods, such as the well-established Maxwell, Garnett and Bruggeman formalisms, applicable to nanocomposite materials. These formalisms are extended in order to accommodate the non-zero size of the component particles. Theoretical results are established for isotropic chiral and uniaxial dielectric homogenized nanocomposites.

\section{References}

[1] I. Freestone, N. Meeks, M. Sax, and C. Higgitt, "The Lycurgus cup - A Roman nanotechnology," Gold Bull. 40, 270-277 (2007).

[2] L. Campbell and W. Garnett, The Life of James Clerk Maxwell, Macmillan, London, UK, (1882).

[3] M. Dienerowitz, M. Mazilu, and K. Dholakia, "Optical manipulation of nanoparticles: a review," J. Nanophoton. 2, 021875 (2008) [doi:10.1117/1.2992045].

[4] M. A. Noginov, "Compensation of surface plasmon loss by gain in dielectric medium," $J$. Nanophoton. 2, 021855 (2008) [doi: 10.1117/1.3073670].

[5] K. A. Serrels, E. Ramsay, P. A. Dalgarno, B. D. Gerardot, J. A. O'Connor, R. H. Hadfield, R. J. Warburton, and D. T. Reid, "Solid immersion lens applications for nanophotonic devices," J. Nanophoton. 2, 021854 (2008) [doi: 10.1117/1.3068652].

[6] Z. Gaburro, "Photonic energy lifters and event horizons with time-dependent dielectric structures," J. Nanophoton. 2, 021853 (2008) [doi:10.1117/1.3054303].

[7] M. W. Vogel and D. K. Gramotnev, "Optimization of plasmon nano-focusing in tapered metal rods," J. Nanophoton. 2, 021852 (2008) [doi:10.1117/1.3046689].

[8] M. A. Vincenti, V. Petruzzelli, A. D’Orazio, F. Prudenzano, M. J. Bloemer, N. Akozbek, and M. Scalora, "Second harmonic generation from nanoslits in metal substrates: applications to palladium-based $\mathrm{H}_{2}$ sensor," J. Nanophoton. 2, 021851 (2008) [doi:10.1117/1.3005971].

[9] T. G. Mackay, "On extended homogenization formalisms for nanocomposites," $J$. Nanophoton. 2, 021850 (2008) [doi:10.1117/1.2982931]. 